\title{
Robot-Assisted Therapy Influence on Kinematic and EMG Activity in the Upper Limb for Children with Cerebral Palsy
}

Authors:

S. Raouafi ${ }^{a^{*}}$, M. Raison ${ }^{b}$, S. Achiche ${ }^{b}$

Affiliations:

a Institute of Biomedical Engineering, École Polytechnique de Montréal, QC, Canada

b Department of Mechanical Engineering, Machine Design Section, École Polytechnique de Montréal, Canada

* Corresponding author:

CRME - Research Center, Office GR-123

5200, East Bélanger Street

H1T 1C9, Montréal, QC, Canada

Tél. : +1 (514) 374-1710 \# 8189

Email address: sana.raouafi@polymtl.ca 


\begin{abstract}
AIM To develop an index for quantitative assessment of the upper limb motor function in children with cerebral palsy before and after robot-assisted therapy.

METHOD An upper limb motor function index was developed using kinematic, surface electromyography and three-axis inertial measurements unit data collected from 15 children with cerebral palsy $(\mathrm{CP})$ and 15 typically developed children. Children with $\mathrm{CP}$ underwent 18 robot-assisted therapy sessions with the REAplan device. All children were evaluated, using kinematic data from the REAplan, electromyography and three-axis inertial measurements unit readings from its accelerometer. A principal component analysis was conducted to produce an evaluation index, which is able to detect the deviation from the upper limb motor function of typically developing children group. Children with $\mathrm{CP}$ were evaluated twice before and after the intervention with Box and Blocks test and Finger-To-Nose test. The discriminative and concurrent validity of the upper limb motor function index were investigated.
\end{abstract}

RESULTS The upper limb motor function index was higher in children with $\mathrm{CP}$ post therapy $(p<0.001)$. Finger-To-Nose test values improved after robot-assisted therapy $(p<0.03)$. A weak but positive correlation was observed between upper limb motor function index and clinical tests $(\rho=0.012, p=0.95$ and $\rho=0.13, p=0.54$ for Box and Blocks test and Finger-To-Nose test respectively).

INTERPRETATION The upper limb motor function index successfully differentiated between the typically developing children and children with $\mathrm{CP}$ and was effective in assessing the improvement of the upper limb motor function after robot-assisted therapy. The upper limb motor function index could be extended to assess and monitor rehabilitation therapies of other populations, such as those with stroke and Parkinson's disease. 
What this paper adds:

- The upper limb motor function index offers an objective alternative to the clinical scales.

- The upper limb motor function index can show whether there was any significant change in kinematic and electromyography activity in the upper limb after intervention.

- The REAplan improved proximal movement patterns in children with cerebral palsy. 


\begin{tabular}{|c|c|}
\hline \multicolumn{2}{|c|}{ Abbreviations } \\
\hline $\mathbf{A A C}$ & Average Amplitude Change \\
\hline $\mathrm{ACC}$ & Accelerometer \\
\hline AHA & The Assisting Hand Assessment \\
\hline $\mathbf{A R}$ & Auto Regression coefficient \\
\hline BBT & Box and Block Test \\
\hline $\mathbf{C C}$ & Cepstrum Coefficients \\
\hline $\mathbf{C P}$ & Cerebral Palsy \\
\hline FMA & Fugl-Meyer Assessment \\
\hline EMG & Electromyography \\
\hline FTN & Finger-To-Nose test \\
\hline GDI & Gait Deviation Index \\
\hline IEMG & Integrated EMG \\
\hline IMU & Inertial Measurement Unit \\
\hline IMU-ACC & IMU readings from accelerometer \\
\hline MAS & The Modified Ashworth Scale \\
\hline MAUULF & The Melbourne Assessment of Unilateral Upper Limb Function \\
\hline MFL & Maximum fractal length \\
\hline MSS & Mean Spike Slope \\
\hline MU & Motor Unit \\
\hline PCA & Principal Component Analysis \\
\hline QUEST & The Quality of Upper Extremity Skills Test \\
\hline Robot-AT & Robot-Assisted Therapy \\
\hline SM2 & 2nd spectral moment \\
\hline $\mathbf{U L}$ & Upper Limb \\
\hline
\end{tabular}


ULMFI Upper limb motor function index

WL Waveform Length

ZC Zero Crossing 


\section{Introduction}

Cerebral palsy $(\mathrm{CP})$ is a group of neurodevelopmental disorders caused by a static insult to the developing brain and affecting movement and posture [1,2]. It is also the most prevalent type of physical disability in children [3]. Upper limb (UL) dysfunction in particular, is one of the most important disabling symptoms and has a major negative effect on children's quality of life [4].

Although CP is a lifelong disability, there are a variety of therapeutic interventions used to improve functional abilities, treat spasticity-related pain and impact children's quality of life. Recently, robotic rehabilitation devices were developed and employed for the pediatric population with neuromotor disorders [5]. Research studies have shown that the use of robotic rehabilitation devices can offer a great improvement in UL motor function compared to conventional therapies $[6,7]$.

To determine the amount of clinically important change after these interventions and decide the patient's robotic rehabilitation needs in the future, the evaluation of UL motor function plays an important role. In most of these studies, the assessment of UL motor function improvement was guided by clinical scales. A variety of assessment tools were used [8], the Fugl-Meyer Assessment (FMA) [9]; the Modified Ashworth Scale (MAS) [10], the Quality of Upper Extremity Skills Test (QUEST) [11], the Melbourne Assessment of Unilateral Upper Limb Function (MAUULF)[12], the Assisting Hand Assessment (AHA)[13] and the Box and Block Test (BBT) [14]. Despite having proved their validity in assessing UL motor function, these scoring systems are still considered a descriptive and subjective evaluation. Moreover, these scoring systems rely on the clinicians' expertise, leading to non-consideration of pathological mechanisms, limited accuracy and an underestimation of child's actual performance [15-17].

On the other hand, few studies have suggested testing UL motor function using kinematic, kinetic and dynamic data, before, during and after the Robot-AT. According to the metaanalysis of V. Falzarano et al. [5], most of these studies presented a clinical application with kinematic outcome measures. In five clinical protocols, the kinematic values smoothness and motor performance - improved significantly in the training group compared to the control group. Gilliaux et al. [18] used the REAplan [19] to compare 
robotic rehabilitation with traditional therapy in children with CP. Greater smoothness ( $p$ $<0.01)$ and manual dexterity $(p<0.04)$ were observed in the training group when compared to the control group. Two other studies using the same device; Armeo Spring system, concluded that significant improvements were present in terms of UL kinematics such as movement duration, velocity of movement execution, smoothness and Number of Unit Number [20, 21].

Quantitative electromyography (EMG) analysis has been widely used in pattern recognition, rehabilitation training and motor control analysis. To the knowledge of the authors, only a few recent studies used EMG features to assess the effectiveness of robotAT. For example, Zadnia et al. [22] showed that Shannon entropy could provide an accurate visual biofeedback for reduction of spasticity in patients with a stroke. Another research study found that implementing an EMG-based model of muscle health in a rehabilitative elbow brace has the potential of assessing patients' recovery from Musculoskeletal (MSK) elbow trauma [23]. Some authors advised to incorporate EMGmeasurements for the assessment of UL movement characteristics in children with CP [24]. However, no study has examined effectiveness of assistive technology interventions for $\mathrm{CP}$ children with kinematic and EMG measurements.

In this research paper, an index for quantitative assessment of the upper limb motor function in children with $\mathrm{CP}$ after robot-AT is proposed, based on fused data from kinematic, EMG and inertial measurements unit readings from accelerometer (IMU-ACC) measurements. A group of typically developing (TD) children was included representing the normal pattern of the task performance. The upper limb motor function index (ULMFI) was developed using a Euclidean distance similar to the GDI [25].

We hypothesized that robot-AT would increase ULMFI values in children with CP and that levels of muscle activation would be closer to those of TD children. 


\section{Methods}

The methods' section is divided as follows:

First, we will present participants and experimental protocol. Second, we will describe the strategy of feature extraction and selection, and finally we will illustrate all steps of data analysis including the concurrent and discriminative validity.

\section{Participants and experimental protocol}

$15 \mathrm{CP}$ children (mean age: 9 y 5 mo, interquartile range [IQR] 8:10y) and 15 TD children (mean age: 8 y 2 mo, [IQR] 6-11y) were recruited in the data collection experiment. The inclusion criteria consist of: a history of $\mathrm{CP}$, a maximum age of 14 years and the ability to understand or perform the given tasks. Furthermore, TD children needed to not having had a history of joint or neurological disorders. Among the exclusion criteria were botulinum toxin injections within six months before measurements or previous orthopedic surgery in the UL for CP children. CP patients were recruited from a school for children with physical disabilities (École Victor-Doré, Montréal, Qc, Canada). The study was approved by the Research Ethics Boards of Ste. Justine Hospital (protocol code: 2017-1458). The informed consent was obtained from all subjects prior to their participation. The patients' characteristics are described in Table 1.

Table 1 Demographic characteristics.

\begin{tabular}{lll}
\hline & Cerebral palsy & Typically developing \\
\hline $\mathrm{n}$ & 15 & 15 \\
Age, y: mo (IQR) & $9: 5(8: 0-10: 0)$ & $8: 2(6: 0-11: 0)$ \\
Gender, males/females & $8 / 7$ & $10 / 5$ \\
Box and Block test, mean & $21.85(10.40)$ & - \\
Coordination proximal, mean & $15.23(6.43)$ & - \\
MACS levels & I: 3 & - \\
& II: 6 & - \\
& III: 5 & - \\
& IV: 1 & - \\
\hline
\end{tabular}

IQR, interquartile range; MACS, Manual Ability Classification System

In this study, every CP child underwent a 10-week training with the end-effector REAplan robot [26] with two weekly sessions. Each session lasted for $45 \mathrm{~min}$. During each session 
using REAplan, children performed four standard tasks; two unidirectional tasks (a target task and a free Amplitude task) and two geometrical tasks (circle task and a square task), with the supervision of their occupational therapist. They were evaluated using REAplan over two sessions: before the intervention and at the end of the 10-week program. Before and after the intervention, occupational therapists adopted two clinical scales to assess the motor function of the UL of the CP children, namely the Box and Block test (BBT; to measure unilateral gross manual dexterity) and the Finger-To-Nose test (FTN; to quantify the degree of impaired coordination).TD children were evaluated once using the REAplan. Datasets from two CP subjects were excluded from further analysis due to: 1 . Sensor data corrupted by large unexpected noises; 2. Hardware failure during the data acquisition. Furthermore, two TD children were excluded due to missing data from two UL kinematic data during evaluation with REAplan.

\section{Strategy of feature extraction and selection}

\section{Kinematic parameters}

UL kinematics were computed from each task. For the Free Amplitude task, the smoothness, speed and straightness indices were computed. For the Target task, an inaccuracy index was added. For the Square and Circle tasks, the speed, inaccuracy and smoothness indices were computed.

\section{EMG and IMU-ACC features}

sEMG and IMU-ACC data were collected from wearable sensors (Trigno, Delsys Inc., Natick, MA, USA). Each sensor included information from both muscle activation, via surface EMG sensors, and position by triaxial ACC. Eight sensors were attached to the surface of the skin of the anterior deltoid, lateral deltoid, posterior deltoid, biceps brachii, triceps brachii, infraspinatus, brachialis and brachioradialis, according to the guidelines of the SENIAM project [27]. Forty sEMG features and six IMU-ACC features were extracted from each EMG segment and accelerometer signal. More details about the feature extraction method used in this paper could be found in the author's recently published 
classification model for the assessment of UL motor function using data from EMG and IMU-ACC presented in [28].

\section{Feature selection}

From our previous study [28], the best accuracy to differentiate between TD children and children with CP pre- and post-Robot-AT was obtained during the circle task. Each child had to draw a circle with a 4-cm radius using REAplan. Thus, in the current study only UL kinematics from the circle task will be analyzed. Consequently, a total of 12 extracted features were used for further analysis. The Kaiser-Meyer-Olkin (KMO) measure was used to reduce the set of 12 features in preparation for factor analysis. Bartlett Test of Sphericity at $426.158, p=0.000$ and $\mathrm{KMO}=0.87$ indicated that the data were appropriate for the purpose of PCA. From the 12 features, only the velocity did not meet the criteria of measurement of sampling adequacy $(=>0.50) .11$ features were then included: inaccuracy, smoothness, Spectral Moment 2 (SM2), Integrated EMG (IEMG), Average Amplitude Change (AAC), Zero Crossing (ZC), Multiple Hamming Window (MHW), the fourth Coefficient of Cepstral Analysis (CC4), Maximum Fractal Length (MFL), Mean Spike Slope (MSS) and the mean of the magnitude of acceleration (mean IMU-ACC).

\section{Data analysis}

\section{Calculation of similarity indices}

Among all extracted features, eight EMG and one IMU-ACC features provided enough information to distinguish between healthy children and children with $\mathrm{CP}$ pre-robot-AT and post-robot-AT [28]. Each EMG and IMU-ACC feature can be arranged as an 8-element vector for all channels. For each feature vector, we first calculated the Euclidean distances between any given $\mathrm{CP}$ child $\alpha$ and all TD children. Then we selected the maximal distance as a similarity index which can be described as follows:

$\mathrm{d}^{\alpha, \mathrm{TD}}=\max \left(\left\|c^{\alpha}-\mathrm{c}^{\beta_{\mathrm{j}}}\right\|\right)$

where:

c refers to a feature vector 
$\alpha$ refers to a $\mathrm{CP}$ child

$\beta_{\mathrm{j}}$ refer to TD child; $\mathrm{j}=\{1, \ldots, \mathrm{n}\}, \mathrm{n}$ is the sample size of TD children.

The minimal distance was used instead of the maximal distance to produce similarity indices for each TD child with respect to the normal reference.

\section{ULMFI generation}

Principal Component Analysis (PCA) [29] was conducted to reduce the feature set into a set of principal dimensions while preserving as much information as possible. Since the principal component transformation is not scale invariant, each variable was normalized by subtracting the mean and dividing by the standard deviation using the TD group. We calculated the ULMFI using a distance metric similar to the GDI [25]. The m retained principal components were then considered as a data sample matrix with each row representing an m-dimensional feature vector for each subject. Here, the purpose of the PCA was not the reduction of the feature space but rather transforming the original possibly correlated features into a simpler data set with unrelated variables. The new components will be used to define the ULMFI score and avoid any overlap between the original parameters used to compute the deviation from the TD group.

Given the Euclidean distance between a CP subject $\alpha$ and the average TD group, the raw ULMFI for subject $\alpha$ is defined as:

$\mathrm{ULMFI}_{\text {raw }}^{\alpha}=\ln \left(\mathrm{d}^{\alpha, \mathrm{TD}}\right)$

Where $d^{\alpha, T D}=\left\|c^{\alpha}-c^{\overline{T D}}\right\| ; c^{\overline{T D}}$ is the average feature component vector of the TD group.

For clinical interpretation purposes [30], the ULMFI for each CP child $\alpha$ was transformed to a scaled version of the TD group (Mean (SD); 100 (10)) as follows:

$$
\begin{aligned}
& \mathrm{zULMFI}_{\text {raw }}^{\alpha}=\frac{\mathrm{ULMFI}_{\text {raw }}^{\alpha}-\mathrm{Mean}_{\left(\mathrm{ULMFI}_{\mathrm{raw}}^{\mathrm{TD}}\right)}}{\mathrm{SD}\left(\mathrm{ULMFI}_{\mathrm{raw}}^{\mathrm{TD}}\right)} \\
& \mathrm{ULMFI}^{\alpha}=100-10 \times \mathrm{zULMFI}_{\text {raw }}^{\alpha}
\end{aligned}
$$

Where ULMFI TD is the raw ULMFI for each subject in TD group. 


\section{Validity}

The concurrent validity of the ULMFI was evaluated by examining its behavior with respect to clinical scales: BBT and FTN tests. The discriminative validity of ULMFI in CP children pre- and post-Robot-AT and TD group was investigated with Kruskal Wallis, Mann-Whitney and paired Wilcoxon tests with Bonferroni correction, by use of kinematic, EMG and IMU-ACC measurements. All statistical analyses were performed using R Statistical Software (version 3.6.1; R Foundation for Statistical Computing platform). 


\section{Results}

At first, we will present results for feature reduction using PCA. Then, differences in ULMFI between TD children and children with CP pre- and post-Robot-AT will be illustrated. Finally, we will show whether there were significant changes in kinematic and EMG activity in the UL post-Robot-AT.

After performing PCA, when load attributes of the 11 features, the first six components explaining more than 98 percent of the total variance were retained for further analysis. Results for analysis are represented using graph of PCA with the first two dimensions (Figure 1 (a)).

安 TD CP pre Robot-AT CP post Robot-AT

(a)

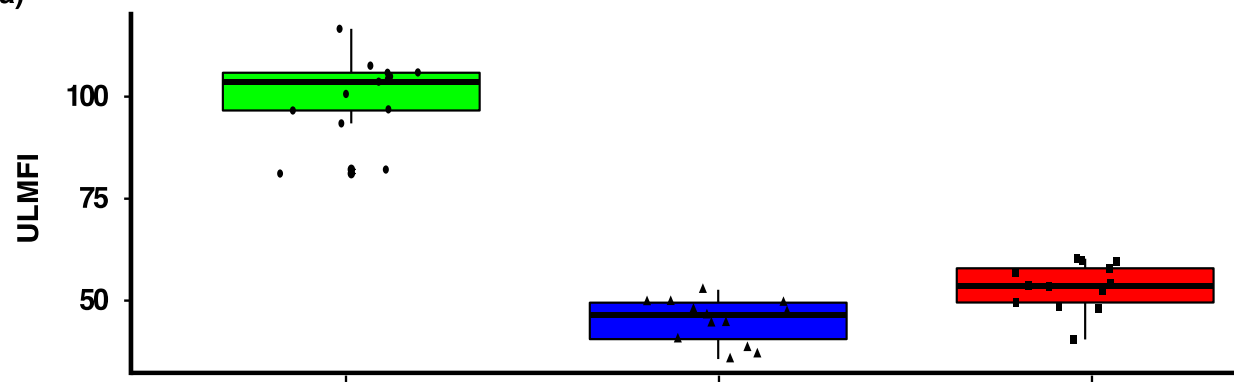

Children conditions

(b)

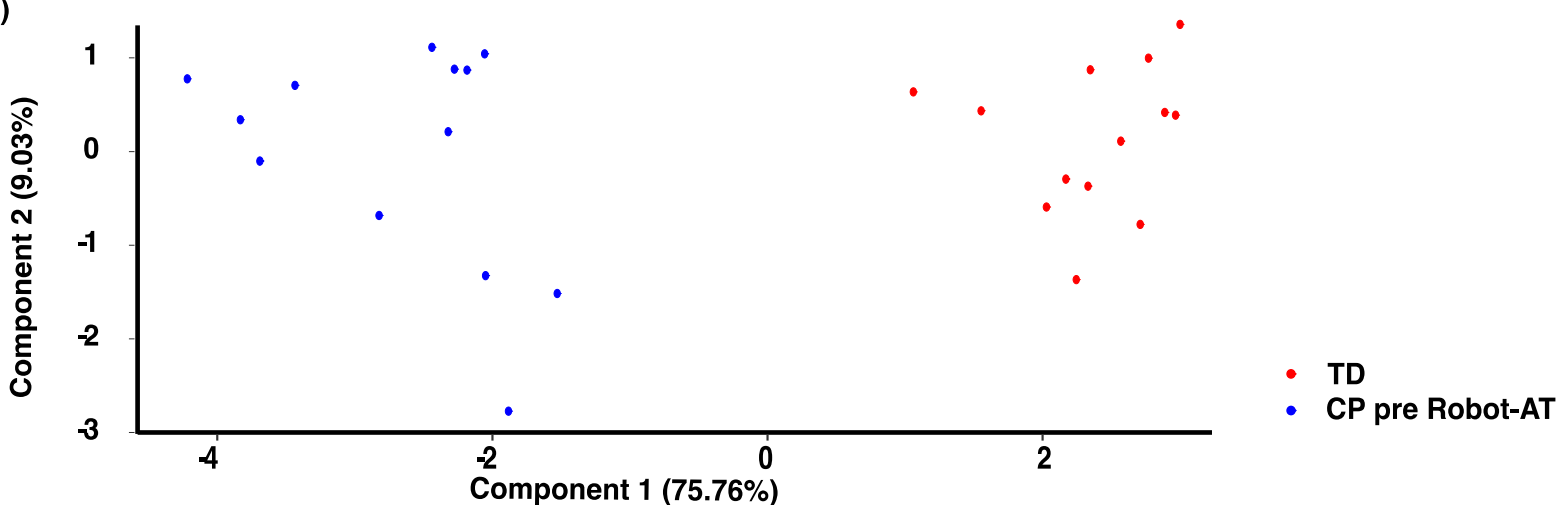

Figure 1 (a) Comparison of the Upper Limb Motor Function Index between Typically Developing (TD) children, children with cerebral palsy pre-Robot-Assisted Therapy; (b) Sample representation using the first two components from Principal Component Analysis (PCA). 
PCA clearly separates TD and CP groups. The six retained components were then employed to generate ULMFI for CP children pre-Robot-AT and TD children. ULMFI for $\mathrm{CP}$ children post-Robot-AT were predicted using the loading matrix of PCA. Pairwise comparisons using Wilcoxon rank sum test revealed significant differences $(p<0.001)$. Figure 1 (b) shows the mean ULMFI for TD children (mean (SD), 100 (10)) and pre-post changes for CP children (44.9 (5.43) and 53.4 (5.73), for CP pre- and post-Robot-AT respectively). The ULMFI improved significantly after Robot-AT. Boxplots in Figure 2 show a distinction between the TD and CP groups, with a closer mean value for each feature in the $\mathrm{CP}$ group post-Robot-AT to those of TD group.

For all EMG and IMU-ACC features, the Kruskal Wallis test revealed significant differences between groups. Results are presented in Table 2 and Figure 2, only features with large effect size will be discussed [31] ( $r=>0.5)$. Results for smoothness and inaccuracy variables are shown in Table 3. 
Table 2 Significant group difference according to the Mann-Whitney $U$ and paired Wilcoxon tests, accompanied with effect sizes r. Only features with significant differences between the comparing groups are reported. Variable definitions are reported in [28].

\begin{tabular}{|c|c|c|c|c|c|}
\hline \multicolumn{6}{|c|}{ TD vs CP children pre-Robot-AT } \\
\hline Feature & Muscle & Mean & Mean & $p$ & $r$ \\
\hline \multirow[t]{3}{*}{$\overline{\mathrm{SM} 2}$} & Biceps & $0.02(0.03)$ & $0.46(0.79)$ & 0.010 & 0.50 \\
\hline & Infraspinatus & $0.04(0.02)$ & $0.19(0.32)$ & 0.031 & 0.42 \\
\hline & Triceps & $0.02(0.01)$ & $0.27(0.49)$ & 0.031 & 0.42 \\
\hline IEMG & Biceps & $0.38(0.30)$ & $1.69(2.01)$ & 0.008 & 0.51 \\
\hline \multirow[t]{3}{*}{$\mathrm{ACC}$} & Lateral deltoid & $0.96(0.01)$ & $0.94(0.01)$ & 0.013 & 0.48 \\
\hline & Brachialis & $0.95(0.02)$ & $0.92(0.03)$ & 0.021 & 0.45 \\
\hline & Posterior deltoid & $1.13(0.04)$ & $1.02(0.07)$ & $<0.001$ & 0.75 \\
\hline \multirow[t]{4}{*}{$\mathrm{AAC}$} & Biceps & $4.40(3.09)$ & $22.68(26.12)$ & 0.016 & 0.47 \\
\hline & Infraspinatus & $7.32(1.71)$ & $13.90(8.06)$ & 0.010 & 0.50 \\
\hline & Triceps & $5.89(2.00)$ & $17.36(17.74)$ & 0.045 & 0.39 \\
\hline & Anterior deltoid & $9.15(5.88)$ & $19.93(14.22)$ & 0.022 & 0.45 \\
\hline \multirow[t]{3}{*}{ MHW } & Biceps & $1.18(2.40)$ & $45.28(79.84)$ & 0.007 & 0.53 \\
\hline & Triceps & $1.12(0.81)$ & $55.43(15.78)$ & 0.029 & 0.43 \\
\hline & Brachioradialis & $4.25(5.50)$ & $18.48(25.10)$ & 0.044 & 0.39 \\
\hline \multirow[t]{3}{*}{ MFL } & Biceps & $-5.80(0.41)$ & $-4.79(1.10)$ & 0.012 & 0.49 \\
\hline & Infraspinatus & $-5.25(0.21)$ & $-4.81(0.55)$ & 0.031 & 0.42 \\
\hline & Triceps & $-5.51(0.29)$ & $-4.91(0.77)$ & 0.031 & 0.42 \\
\hline \multicolumn{6}{|c|}{ TD vs CP children post-Robot-AT } \\
\hline \multirow[t]{2}{*}{$\overline{\mathrm{SM} 2}$} & Biceps & $0.02(0.03)$ & $0.11(0.26)$ & 0.031 & 0.42 \\
\hline & Brachialis & $0.02(0.01)$ & $0.06(0.07)$ & 0.021 & 0.45 \\
\hline IEMG & Brachialis & $0.38(0.21)$ & $0.67(0.35)$ & 0.035 & 0.41 \\
\hline \multirow{3}{*}{$\mathrm{ACC}$} & Infraspinatus & $0.87(0.05)$ & $0.97(0.10)$ & 0.022 & 0.45 \\
\hline & Brachioradialis & $0.95(0.04)$ & $0.98(0.02)$ & 0.034 & 0.41 \\
\hline & Posterior deltoid & $1.13(0.04)$ & $1.13(0.13)$ & 0.019 & 0.46 \\
\hline MHW & Biceps & $1.18(2.40)$ & $5.95(9.56)$ & 0.018 & 0.46 \\
\hline \multirow[t]{2}{*}{ MFL } & Biceps & $-5.80(0.41)$ & $-5.30(0.69)$ & 0.031 & 0.42 \\
\hline & Brachialis & $-5.57(0.67)$ & $-4.88(1.18)$ & 0.016 & 0.47 \\
\hline \multicolumn{6}{|c|}{ CP children pre_Robot-AT vs CP children post-Robot-AT } \\
\hline \multirow[t]{2}{*}{ SM2 } & Biceps & $0.46(0.79)$ & $0.11(0.26)$ & 0.021 & 0.45 \\
\hline & Anterior deltoid & $0.40(0.56)$ & $0.07(0.08)$ & 0.048 & 0.39 \\
\hline \multirow[t]{3}{*}{$\mathrm{ACC}$} & Infraspinatus & $0.93(0.08)$ & $0.97(0.10)$ & 0.014 & 0.48 \\
\hline & Anterior deltoid & $1.00(0.10)$ & $1.10(0.03)$ & 0.006 & 0.54 \\
\hline & Posterior deltoid & $1.02(0.07)$ & $1.13(0.13)$ & 0.008 & 0.52 \\
\hline \multirow[t]{2}{*}{$\mathrm{AAC}$} & Biceps & $22.68(26.12)$ & $8.06(8.16)$ & 0.040 & 0.40 \\
\hline & Anterior deltoid & $19.93(14.22)$ & $10.01(5.89)$ & 0.010 & 0.50 \\
\hline MHW & Anterior deltoid & $79.28(17.32)$ & $3.38(3.22)$ & 0.040 & 0.40 \\
\hline \multirow[t]{3}{*}{ MFL } & Biceps & $-4.79(1.10)$ & $-5.30(0.69)$ & 0.048 & 0.39 \\
\hline & Brachialis & $-5.29(0.76)$ & $-4.88(1.18)$ & 0.040 & 0.40 \\
\hline & Anterior deltoid & $-4.59(0.68)$ & $-5.03(0.43)$ & 0.017 & 0.47 \\
\hline
\end{tabular}


$\mathrm{CC} 4$ Infraspinatus $0.41(0.08)$ $0.33(0.10)$ 0.048 0.39

Bonferroni-corrected $\mathrm{p}$-values are reported. Bold type indicates large effect size $(=>0.50)$. TD, Typically Developing; CP pre-Robot-AT, children with cerebral palsy pre-RobotAssisted Therapy; CP post-Robot-AT, children with cerebral palsy post-Robot-Assisted Therapy; AAC and MHW are in [uV].

Figure 2 Boxplots of similarity indices for kinematic, EMG and IMU-ACC parameters

(a) Imprecision

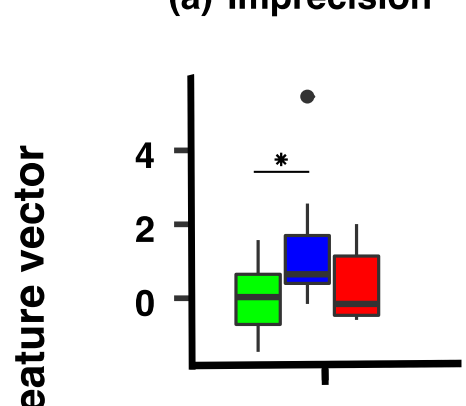

(e) AAC (mV)

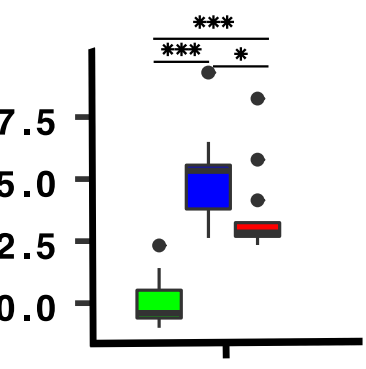

(b) Smoothness

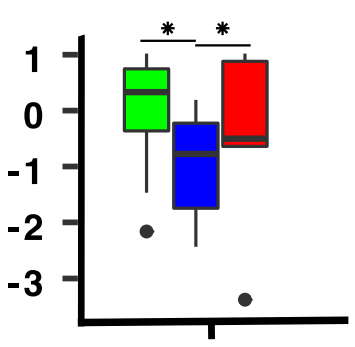

(f) ZC
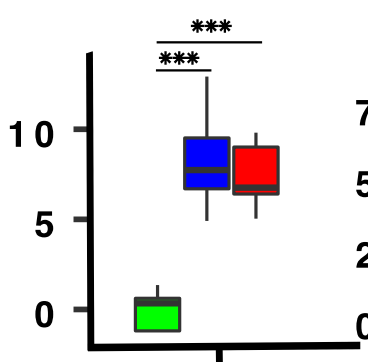

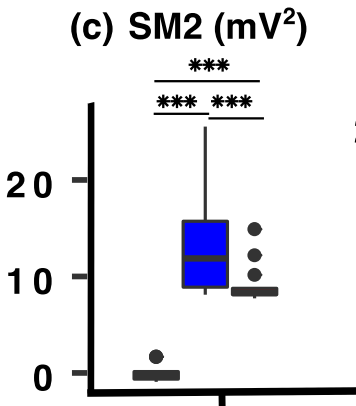

(g) MHW (mV)

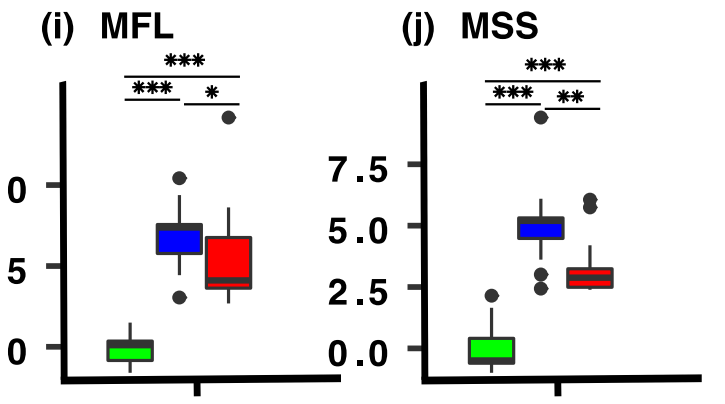

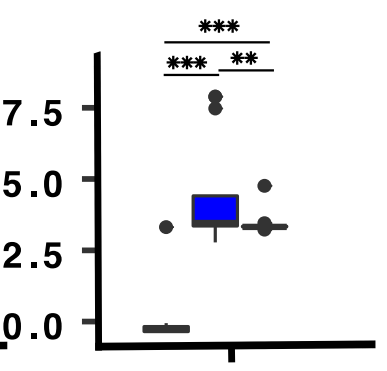

(k) $\mathrm{ACC}(\mathrm{g})$

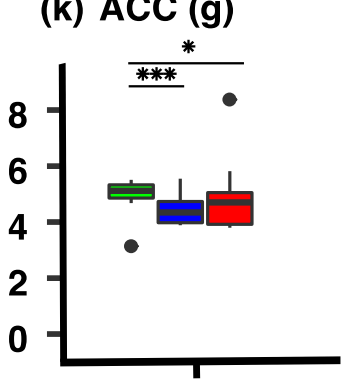

(d) IEMG (mV.s)

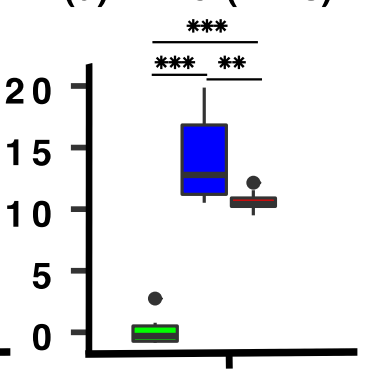

(h) $\mathrm{CC}$

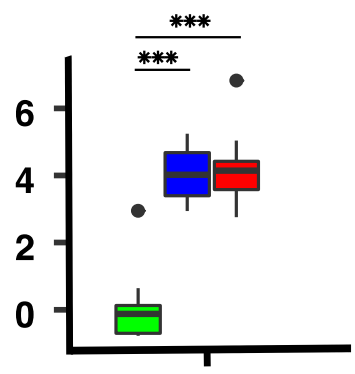

\section{Features}

for Typically Developing (TD) children, children with cerebral palsy pre-Robot-Assisted Therapy (CP pre-Robot-AT) and CP post-Robot-AT (children with cerebral palsy postRobot-Assisted Therapy. Variable definitions are reported in [28]. 
Table 3 Significant group difference according to the Mann-Whitney $U$ and paired Wilcoxon tests, accompanied with effect sizes $r$ for smoothness and inaccuracy variables.

\begin{tabular}{lllll}
\hline \multicolumn{5}{c}{ TD vs CP children pre-Robot-AT } \\
\hline Variable & Mean & Mean & $p$ & $r$ \\
\hline Smoothness & $0.68(0.07)$ & $0.61(0.06)$ & 0.010 & 0.49 \\
Inaccuracy & $0.52(0.16)$ & $0.72(0.24)$ & 0.020 & 0.46 \\
\hline \multicolumn{5}{c}{ TD vs CP children post-Robot-AT } \\
\hline Smoothness & $0.68(0.07)$ & $0.66(0.09)$ & 0.661 & 0.09 \\
Inaccuracy & $0.52(0.16)$ & $0.56(0.15)$ & 0.540 & 0.12 \\
\hline \multicolumn{5}{c}{ CP children pre_Robot-AT vs CP children post-Robot-AT } \\
\hline Smoothness & $0.61(0.06)$ & $0.66(0.09)$ & 0.054 & 0.37 \\
Inaccuracy & $0.72(0.24)$ & $0.56(0.15)$ & 0.033 & 0.42 \\
\hline
\end{tabular}
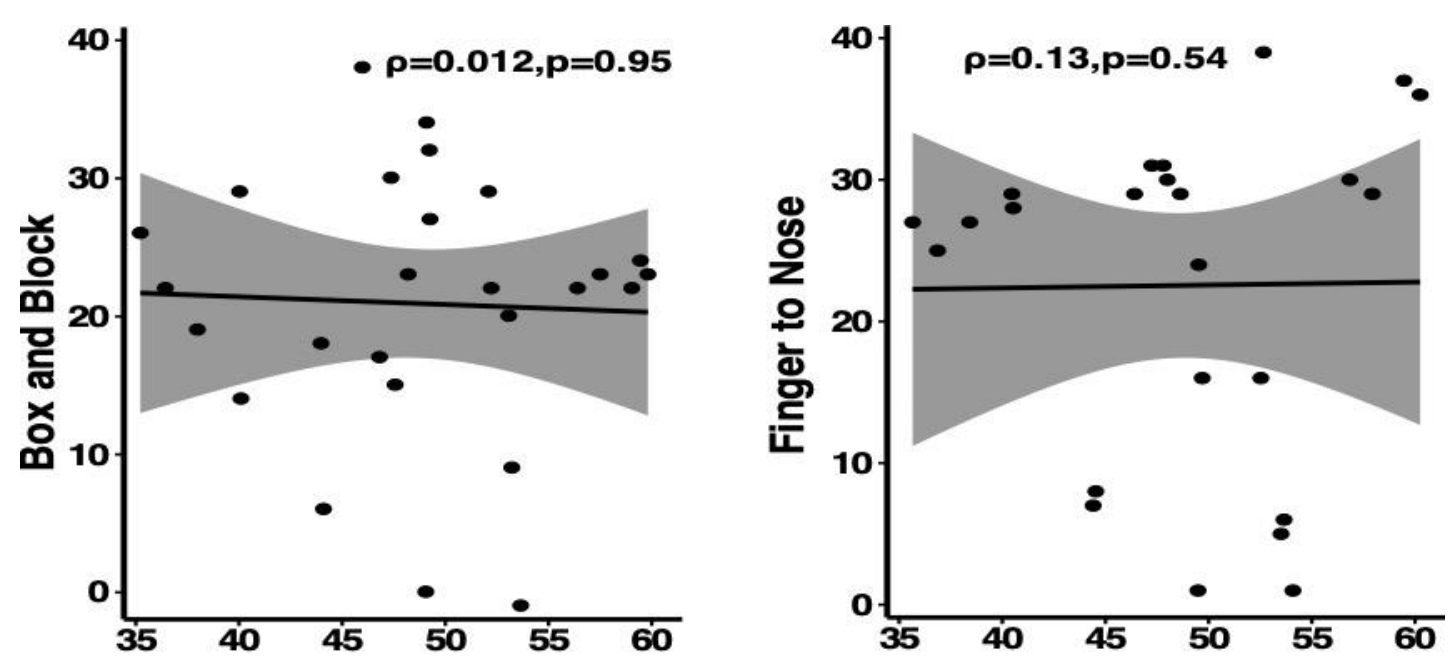

ULMFI

Figure 3 Spearman's rank correlation $(\rho)$ between clinical tests and Upper Limb Motor Function Index (ULMFI).

For CP group pre- and post-Robot-AT, Figure 3 shows a weak but a positive correlation between ULMFI and clinical tests $(\rho=0.012, p=0.95$ and $\rho=0.13, p=0.54$ for BBT and FTN respectively). Wilcoxon rank sum test revealed significant differences before and after Robot-AT for FTN ( $p=0.03)$ but not for BBT ( $p=0.57)$. 


\section{Discussion}

The aim of this study was to assess the effectiveness of Robot-AT in children with $\mathrm{CP}$ using a quantitative ULMFI. The ULMFI was developed using a distance metric similar to the GDI [25]. The discriminative validity of the ULMFI was established in CP children pre-Robot-AT and TD group by use of kinematic, EMG and IMU-ACC variables. The concurrent validity was determined by comparing the ULMFI to clinical assessments in $\mathrm{CP}$ children pre- and post-Robot-AT.

The ULMFI was developed based on two kinematic parameters from the REAplan, eight EMG features and mean IMU-ACC. In our recent study, the eight EMG features were the top ranked features among each category of sEMG features. Moreover, adding mean IMUACC provided the best classification accuracy in distinguishing TD, children with $\mathrm{CP}$ preand post-Robot-AT during the circle task [28]. The results support our hypothesis that Robot-AT would increase ULMFI values in children with CP and that levels of muscle activation would be closer to those of TD children (Figure 1 (b)) and Figure 2). These findings are in accordance with recent studies that reported that Robot-AT offers a great improvement in UL motor function and could be considered as a promising intervention for children with $\mathrm{CP}[6,18,21]$.

Results reported Figure 2 and Table 2 showed that some parameters appear to have a great potential in distinguishing between $\mathrm{CP}$ and TD children. The posterior deltoid muscle showed higher mean IMU-ACC values for TD group and CP post-Robot-AT in comparison to CP pre-Robot-AT (Table 2). The decreased mean IMU-ACC may be caused by poor selective motor control [32] during shoulder horizontal extension for circle task. Several studies proved that this parameter can be improved with rehabilitation therapies [33, 34], which is the case for CP children post-Robot-AT in the current study.

Results on the significance of differences between healthy and pathological muscles showed a general trend of higher values in the biceps and infraspinatus muscles for the SM2, IEMG, AAC and MHW parameters in CP group (Figure 2 (c), (d), (e) and (g) respectively). This is consistent with the necessity of the activation of the elbow and shoulder muscles to perform the extension/flexion and external rotation movements of the shoulder during the circle task. SM2 feature was different in triceps and infraspinatus 
muscles as well, with higher activation in pre-Robot-AT group. This is in accordance with Prosser et al. [35] who reported high values of the time-frequency domain features in all muscles for the $\mathrm{CP}$ group during gait cycle. For $\mathrm{CP}$ group, AAC exhibited higher values in infraspinatus, triceps and anterior deltoid muscles than TD group. For MHW, higher activation was observed in biceps, triceps and brachioradialis muscles in pre-Robot-AT group. The results of the paired difference tests suggest that there is more MU (Motor Unit) recruitment in pathological muscles compared to healthy muscles, in terms of signal energy, multi-window and information complexity of the EMG signals.

There was a general trend showing a higher mean IMU-ACC in anterior and posterior deltoid muscles and lower AAC in biceps muscle for children post-Robot-AT. The AAC feature is similar to the waveform length (WL) feature but averages the WL over the time segment. This feature provides information on the waveform complexity in each segment and indicates the degree of variations in EMG signals [36]. Increase in EMG signal complexity as seen by AAC indicates increase in activation level of the muscles. Results indicate that higher levels of activation of EMG features were detected in CP pre-RobotAT compared to TD group [37]. Neurological damage in children with CP results in recruiting a higher number of motor units and the need to stabilize the joints, to achieve the same movement as the TD group [38]. This is in agreement with Haddara et al. [39] who found that time-domain metrics were higher in patients recovering from MSK elbow trauma versus the healthy population. The results of this study suggested that children in pre-Robot-AT have progressed in their therapy. This was particularly seen in the mean IMU-ACC of the anterior and posterior deltoid muscles, and the AAC of the anterior deltoid muscle activity, which tended to be similar to those of TD children.

A further finding in this research study was the improved smoothness after Robot-AT (Figure 2 (b)), which is similar to the observations made by several recent studies $[5,6$, $18]$.

The ULMFI exhibited a non-significant but a positive correlation with BBT and FTN (Figure 3). This positive correlation may explain the proximal motor control of the UL, involved in BBT and FTN. The poor correlation observed in this study may be partly due to the relatively small sample size or to the fact that kinematic, EMG and IMU-ACC 
measurements are able to detect specific changes in UL motor function after intervention that conventional scales could not quantify. Motor function improved after Robot-AT according to FTN which is consistent with the improved EMG and the mean IMU-ACC parameters for the proximal muscles that controls the shoulder [40]. However, other clinical assessments may be more appropriate and should be administrated to depict a real improvement in these muscles, such as the proximal upper limb section in the FMA.

We propose the ULMFI as a quantitative assessment for children with CP after Robot-AT. Higher activation in proximal muscles around the shoulder could be due to the increased MU recruitment and a compensatory movement strategy. CP children post-Robot-AT tended to exhibit kinematic, EMG and IMU-ACC signals closer to those of TD group.

One limitation of this study was the relatively small sample size $(n=26)$ which may affect the reliability of statistical results. Due to this small sample, the parametric assumptions were not met. Therefore, nonparametric tests were carried out instead of parametric tests. Future studies may therefore include a larger sample of CP children to validate the quantitative assessment index proposed in this study. Another consideration is that all tasks in the evaluation process are made in a small workspace without regard to the patient's specific characteristics and abilities. In this context, evaluation tasks may be less relevant, and results may be misleading. A patient-specific evaluation task and an individualized trajectory should be designed for the REAplan.

\section{Conclusion}

The objective of this study was to develop an index for quantitative assessment of the upper limb motor function in children with cerebral palsy before and after intervention using the REAplan.

The main results showed that the ULMFI was higher in children with cerebral palsy post therapy $(p<0.001)$. FTN values improved after therapy $(p<0.03)$. A weak but positive correlation was observed between ULMFI and clinical tests $(\rho=0.012, p=0.95$ and $\rho=0.13$, $p=0.54$ for BBT and FTN respectively). 
These results showed that the ULMFI could successfully differentiate between the typically developing group and children with cerebral palsy and was effective in assessing the improvement of upper limb motor function post therapy.

The ULMFI could be extended to assess and monitor rehabilitation therapies of other populations, such as those with stroke and Parkinson's disease.

\section{Acknowledgments}

We gratefully acknowledge the contribution of the Canada Foundation for Innovation (CFI). The authors would like to thank the patients who participated in this study as well as the occupational therapists" team at "École Victor-Doré" for their collaboration. 


\section{References}

[1] M. Bax et al., "Proposed definition and classification of cerebral palsy, April 2005," vol. 47, no. 8, p. 571-576, 2005.

[2] P. Rosenbaum et al., "A report: the definition and classification of cerebral palsy April 2006," vol. 109, p. 8-14, Feb 2007. [En ligne]. Disponible: https://www.ncbi.nlm.nih.gov/pubmed/17370477

[3] S. o. C. P. i. Europe, "Surveillance of cerebral palsy in Europe: a collaboration of cerebral palsy surveys and registers. Surveillance of Cerebral Palsy in Europe (SCPE)," vol. 42, nº. 12, p. 816-24, Dec 2000. [En ligne]. Disponible: https://www.ncbi.nlm.nih.gov/pubmed/11132255

[4] A. Colver et al., "Self-reported quality of life of adolescents with cerebral palsy: a cross-sectional and longitudinal analysis," vol. 385, nº. 9969, p. 705-16, Feb 21 2015. [En ligne]. Disponible: https://www.ncbi.nlm.nih.gov/pubmed/25301503

[5] V. Falzarano et al., "Devices and Protocols for Upper Limb Robot-Assisted Rehabilitation of Children with Neuromotor Disorders," vol. 9, no . 13, p. 2689, 2019.

[6] A. Meyer-Heim et H. J. van Hedel, "Robot-assisted and computer-enhanced therapies for children with cerebral palsy: current state and clinical implementation," vol. 20, no. 2, p. 139-45, Jun 2013. [En ligne]. Disponible: https://www.ncbi.nlm.nih.gov/pubmed/23948688

[7] S. E. Fasoli et al., "Upper limb robotic therapy for children with hemiplegia," vol. 87, $\mathrm{n}^{\mathrm{o}}$. 11, p. 929-36, Nov 2008. [En ligne]. Disponible: https://www.ncbi.nlm.nih.gov/pubmed/18936558

[8] L. V. Wagner et J. R. Davids, "Assessment tools and classification systems used for the upper extremity in children with cerebral palsy," vol. 470, $\mathrm{n}^{\circ} .5, \mathrm{p} .1257-71$, May 2012. [En ligne]. Disponible: https://www.ncbi.nlm.nih.gov/pubmed/21932104

[9] A. R. Fugl-Meyer et al., "The post-stroke hemiplegic patient. 1. a method for evaluation of physical performance," vol. 7, $\mathrm{n}^{\mathrm{o}}$. 1, p. 13-31, 1975. [En ligne]. Disponible: https://www.ncbi.nlm.nih.gov/pubmed/1135616

[10] R. W. Bohannon et M. B. Smith, "Interrater reliability of a modified Ashworth scale of muscle spasticity," vol. 67, nº. 2, p. 206-7, Feb 1987. [En ligne]. Disponible: https://www.ncbi.nlm.nih.gov/pubmed/3809245

[11] C. DeMatteo et al., "The reliability and validity of the Quality of Upper Extremity Skills Test," vol. 13, no. 2, p. 1-18, 1993.

[12] L. M. Johnson et al., "Development of a clinical assessment of quality of movement for unilateral upper-limb function," vol. 36, nº 11, p. 965-73, Nov 1994. [En ligne]. Disponible: https://www.ncbi.nlm.nih.gov/pubmed/7958514 
[13] L. Krumlinde-Sundholm et al., "The Assisting Hand Assessment: current evidence of validity, reliability, and responsiveness to change," vol. 49, nº 4, p. 259-64, Apr 2007. [En ligne]. Disponible: https://www.ncbi.nlm.nih.gov/pubmed/17376135

[14] V. Mathiowetz et al., "Adult norms for the Box and Block Test of manual dexterity," vol. 39, $\mathrm{n}^{\mathrm{o}}$. 6, p. 386-91, Jun 1985. [En ligne]. Disponible: https://www.ncbi.nlm.nih.gov/pubmed/3160243

[15] E. Gajewska, "Narzędzia diagnostyczne do oceny wczesnego rozwoju motorycznego stosowane w fizjoterapii dziecięcej," vol. 20, nº 40, p. 53-58, 2011.

[16] A. P. Tedesco, R. D. Nicolini-Panisson et A. de Jesus, "SHUEE on the evaluation of upper limb in cerebral palsy," vol. 23, nº 4, p. 219-22, Jul-Aug 2015. [En ligne]. Disponible: https://www.ncbi.nlm.nih.gov/pubmed/26327806

[17] M. Penta et al., "The ABILHAND questionnaire as a measure of manual ability in chronic stroke patients: Rasch-based validation and relationship to upper limb impairment," vol. 32, no. 7, p. 1627-34, Jul 2001. [En ligne]. Disponible: https://www.ncbi.nlm.nih.gov/pubmed/11441211

[18] M. Gilliaux et al., "Upper limb robot-assisted therapy in cerebral palsy: a singleblind randomized controlled trial," vol. 29, nº. 2, p. 183-92, Feb 2015. [En ligne]. Disponible: https://www.ncbi.nlm.nih.gov/pubmed/25015650

[19] A. R. technologies, éd.

[20] V. Cimolin et al., "The Armeo spring as training tool to improve upper limb functionality in hemiplegic cerebral palsy: a pilot study," communication présentée à 2016 IEEE 2nd International Forum on Research and Technologies for Society and Industry Leveraging a better tomorrow (RTSI), 2016, p. 1-4.

[21] V. Cimolin et al., "Robot-Assisted Upper Limb Training for Hemiplegic Children with Cerebral Palsy," vol. 31, nº 1, p. 89-101, 2019.

[22] A. Zadnia et al., "Generating the Visual Biofeedback Signals Applicable to Reduction of Wrist Spasticity: A Pilot Study on Stroke Patients," vol. 9, no. 1, p. 15, 2018.

[23] E. Farago et al., "Development of an EMG-Based Muscle Health Model for Elbow Trauma Patients," vol. 19, no . 15, p. 3309, 2019.

[24] E. Jaspers et al., "Three-dimensional upper limb movement characteristics in children with hemiplegic cerebral palsy and typically developing children," vol. 32, $\mathrm{n}^{\mathrm{o}}$. 6, p. 2283-94, Nov-Dec 2011. [En ligne]. Disponible: https://www.ncbi.nlm.nih.gov/pubmed/21862283

[25] M. H. Schwartz et A. Rozumalski, "The Gait Deviation Index: a new comprehensive index of gait pathology," vol. 28, $\mathrm{n}^{\mathrm{o}}$. 3, p. 351-7, Oct 2008. [En ligne]. Disponible: https://www.ncbi.nlm.nih.gov/pubmed/18565753

[26] J. Sapin et al., "Développement du robot de rééducation du membre supérieur REAplan," communication présentée à XLIèmes Journées d'étude de la SORNEST. Technologies innovantes en Médecine Physique et Réadaptation, 2013. 
[27] H. J. Hermens et al., "Development of recommendations for SEMG sensors and sensor placement procedures," vol. 10, nº. 5, p. 361-74, Oct 2000. [En ligne]. Disponible: http://www.ncbi.nlm.nih.gov/pubmed/11018445

[28] S. Raouafi, M. Raison et A. Sofiane, "Modeling the assessment of the upper limb motor function impairment in children with cerebral palsy using sEMG and IMU sensors," p. 748202, 2019.

[29] G. Dallas, "Principal Component Analysis 4 Dummies: Eigenvectors, Eigenvalues and Dimension Reduction," 2013.

[30] J. Y. Zhou et al., "The Pediatric Temporal-spatial Deviation Index: quantifying gait impairment for children with cerebral palsy," Jun 2019. [En ligne]. Disponible: https://www.ncbi.nlm.nih.gov/pubmed/31206183

[31] J. Cohen, "The t test for means. Statistical power analysis for the behavioural sciences," éd: Hillsdale, NJ: Lawrence Erlbaum Associates, 1988.

[32] A. C. Eliasson, A. M. Gordon et H. Forssberg, "Basic co-ordination of manipulative forces of children with cerebral palsy," vol. 33, nº 8, p. 661-70, Aug 1991. [En ligne]. Disponible: https://www.ncbi.nlm.nih.gov/pubmed/1916022

[33] A. C. Eliasson et al., "The Manual Ability Classification System (MACS) for children with cerebral palsy: scale development and evidence of validity and reliability," vol. 48, $\mathrm{n}^{\mathrm{o}}$. 7, p. 549-54, Jul 2006. [En ligne]. Disponible: https://www.ncbi.nlm.nih.gov/pubmed/16780622

[34] R. T. Harbourne, "Accuracy of movement speed and error detection skills in adolescents with cerebral palsy," vol. 93, no. 2, p. 419-31, Oct 2001. [En ligne]. Disponible: https://www.ncbi.nlm.nih.gov/pubmed/11769898

[35] L. A. Prosser et al., "Trunk and hip muscle activity in early walkers with and without cerebral palsy--a frequency analysis," vol. 20, nº. 5, p. 851-9, Oct 2010. [En ligne]. Disponible: https://www.ncbi.nlm.nih.gov/pubmed/20472460

[36] L. J. Bruno, J. Davidowitz et R. F. Hefferline, "EMG waveform duration: A validation method for the surface electromyogram," vol. 2, no . 5, p. 211-219, 1970.

[37] A. Sarcher et al., "Patterns of upper limb muscle activation in children with unilateral spastic cerebral palsy: Variability and detection of deviations," vol. 59, p. 85-93, $112018 . \quad$ [En ligne]. Disponible: https://www.ncbi.nlm.nih.gov/pubmed/30216783

[38] M. G. Feltham et al., "Assessment of neuromuscular activation of the upper limbs in children with spastic hemiparetic cerebral palsy during a dynamical task," vol. 20, $\mathrm{n}^{\mathrm{o}}$. 3, p. 448-56, Jun 2010. [En ligne]. Disponible: http://www.ncbi.nlm.nih.gov/pubmed/19666231

[39] R. Haddara, "Elbow Patients' Data Collection and Analysis: An Examination of Electromyography Healing Patterns," 2016.

[40] F. Fitoussi et al., "Kinematic analysis of the upper limb: a useful tool in children with cerebral palsy," vol. 15, $\mathrm{n}^{\mathrm{o}}$. 4, p. 247-56, Jul 2006. [En ligne]. Disponible: https://www.ncbi.nlm.nih.gov/pubmed/16751732 
